Die Genossen Longo und Spano fragen nach der Meinung der sowjetischen Genossen zu ihrer Entscheidung, den [Rück-]Weg von Moskau über Budapest zu nehmen, um sich mit den ungarischen Führern zu treffen.

Gen. Chruschtschow sagt, dass nach seiner Ansicht eine solche Reise sehr nützlich wäre.

Aufzeichnung des Gesprächs von D. Schewljagin

Nicht korrigiertes Stenogramm

RGANI, fond. 52, opis' 1, delo 568, Bl. 43-57

\title{
19. Gespräch Chruschtschows mit der Regierungsdelegation der Volksrepublik Bulgarien am 16. Februar 1957
}

Am Gespräch nahmen teil von bulgarischer Seite der Erste Sekretär des ZK der Kommunistischen Partei Bulgariens Todor Shiwkow, der Vorsitzende des Ministerrates Bulgariens Anton Jugow, der erste Stellvertreter des Vorsitzenden des Ministerrates Georgij Tschankow, der erste Stellvertreter des Vorsitzenden des Ministerrates und Sekretär des Bauernvolksbundes Georgi Trajkow, der Stellvertreter des Vorsitzenden des Ministerrates und Minister für Handel Rajko Damjanow, der Stellvertreter des Vorsitzenden des Ministerrates und Minister für Bildung und Kultur Wylko Tscherwenkow, der Sekretär des ZK der Kommunistischen Partei Bulgariens Entscho Stajkow, der Außenminister Karlo Lukanow, der außerordentliche und bevollmächtigte Botschafter der Volksrepublik Bulgarien in der UdSSR Ljuben Gerasimow.

Von sowjetischer Seite nahmen am Gespräch [außer Chruschtschow] teil der Außenminister der UdSSR A. A. Gromyko, der erste Stellvertreter des Außenministers der UdSSR N. S. Patolitschew, der außerordentliche und bevollmächtigte Botschafter der UdSSR in Bulgarien Ju. K. Prichodow, das Kollegiumsmitglied des MID der UdSSR I. K. Samtschewskij, der Stellvertreter des Abteilungsleiters beim ZK der KPdSU I. T. Winogradow.

Nach den gegenseitigen Begrüßungen sagte Gen. Chruschtschow, dass wir gegenwärtig alle unter dem Eindruck des soeben zu Ende gegangenen Plenums des ZK der KPdSU stehen. Da die Beschlüsse des Plenums veröffentlicht worden sind, werde ich darauf nicht eingehen. Außer zu den Fragen, über die in der Presse berichtet worden ist, gab es auf dem Plenum im Zusammenhang mit der Antwort des ZK des Bundes der Kommunisten Jugoslawiens (BdKJ) ${ }^{360}$ auf unsern Brief auch noch ein Gespräch über Jugoslawien. Wir wollen, dass die Beziehungen zu Jugoslawien sich verbessern. Doch ob sie sich verbessern, das hängt in erster Linie von den Jugoslawen selbst ab. Wir werden keine Zugeständnisse machen. Wir werden auf unseren prinzipiellen Standpunkten bis hin zu einem Bruch bestehen.

Gen. Jugow bemerkt, dass dies richtig ist. 
Gen. Chruschtschow sagte weiter: In einem gewissen Sinne sind wir [Josip Broz] Tito dafür dankbar, dass er mit seiner Rede in Pula 361 unsere Übereinkunft verletzt hat, die in der bulgarischen Erklärung 362 und in anderen gemeinsamen Erklärungen schriftlich fixiert worden ist. Damit hat er uns die Möglichkeit verschafft, seine Position offen kritisieren zu können. Jetzt ist es völlig klar, dass sich die Hoffnungen Titos nicht erfüllt haben, von anderen kommunistischer Parteien unterstützt zu werden. Alle kommunistischen Parteien unterstützten die Haltung Titos nicht. Insbesondere muss man die richtige Haltung der Kommunistischen Partei Bulgariens, der Kommunistischen Partei der Tschechoslowakei und der Partei der Arbeit Albaniens hervorheben. Obgleich sich die albanischen Freunde etwas ereifern. Wir haben mit ihnen darüber gesprochen.

Im Zusammenhang damit bemerkte Gen. Jugow, dass die Jugoslawen die Albaner mit Geringschätzung behandeln, sie sich ihnen gegenüber verächtlich verhalten und aus diesem Grund fühlen sich die albanischen Genossen zu Recht verletzt.

Gen. Chruschtschow sagte: Ja, die Jugoslawen behandeln die Albaner tatsächlich wie eine niedere Rasse. In einem der Gespräche mit Tito habe ich ihm gesagt: Warum bekunden Sie, der ständig für Gleichberechtigung eintritt, so große Verachtung gegenüber den albanischen Genossen; doch wohl nicht etwa deswegen, weil Albanien ein kleines Land ist, denn auch in einem kleinen Land gibt es kluge Leute und begabte Politiker.

In der jugoslawischen Führung nimmt insbesondere [Mijalko] Todorović eine hochmütige und abschätzige Haltung gegenüber den anderen Parteien ein. In einem der Gespräche sagte ich zu [Vukašin] Mićunović, 363 dass Todorović noch mehr Katholik ist als der Papst.

Unlängst habe ich mit Gen. Shiwkow über den in ihrer Zeitung „Trud“ veröffentlichten Artikel gesprochen. Die bulgarischen Genossen haben in diesem Artikel sehr gut die Ergebnisse der wirtschaftlichen Entwicklung Bulgariens und Jugoslawiens verglichen und veranschaulicht. Aus diesem Artikel geht anschaulich hervor, dass die Pro-Kopf-Produktion der Bevölkerung, die in Bulgarien vor der Errichtung der volksdemokratischen Ordnung bedeutend niedriger als bei

361 Tito hatte am 11. November 1956 in Pula unter anderem über die Vorgänge in Polen und Ungarn gesprochen und das dortige Geschehen als aktuell begründeten Kampf des Volkes gegen stalinistische Elemente bezeichnet, der primär nicht den Personenkult, sondern die Systemfragen betreffe. Die UdSSR müsse die gegenüber Jugoslawien abgegebenen Deklarationen von Belgrad (2. Juni 1955) und von Moskau (20. Juni 1956) auch dann gelten lassen, wenn es um andere sozialistische Staaten gehe. Speziell zu den Ereignissen in Polen und Ungarn erklärte Tito, es sei ein fataler Fehler, dem eigenen Volk durch eine fremde Armee Lektionen zu erteilen. Den reaktionären Kräften dort und im Westen werde es so ermöglicht, den Aufstand für ihre Zwecke auszunutzen. Er verurteilte die erste, im Oktober durchgeführte sowjetische Intervention in Ungarn. Die zweite, die ab Anfang November erfolgt war, sei lediglich das „kleinere Übel“ gewesen. Dass es dazu gekommen war, konnte man aber nach seiner Ansicht nicht den Russen allein zum Vorwurf machen.

362 So irrtümlich im Dokument. Offensichtlich ist die Belgrader Deklaration vom 2. Juni 1955 gemeint, in der sich Chruščëv und Bulganin mit dem eigenen, nationalen Weg Jugoslawiens zum Sozialismus einverstanden erklärten. Tito hatte seinerseits dem sowjetischen Lager volle politische Solidarität zugesichert.

363 Jugoslawischer Botschafter in Moskau, dem Chruščëv hohe Wertschätzung und persönliches Vertrauen entgegenbrachte. 
den Jugoslawen war, jetzt aber das Niveau Jugoslawiens bedeutend überholt hat, obwohl sich die Jugoslawen bekanntlich mit ihrem Sonderweg des Aufbaus des Sozialismus brüsten. Den Angaben des Artikels ist zu entnehmen, dass die Jugoslawen nichts Besonderes vorzuweisen haben, womit sie prahlen könnten. Dies ist eine sehr wichtige Methode der Kritik. Wir wollten diesen Artikel sogar in unseren Zeitungen nachdrucken. Doch wir entschieden uns dafür, das nicht zu tun, um den Jugoslawen keinen Vorwand zu liefern, uns zu bezichtigen, wir würden eine antijugoslawische Kampagne organisieren. In einem der Gespräche mit Mićunović habe ich ihm gesagt, dass sie alle Länder belehren, wie man den Sozialismus aufbauen müsse, doch ist bei ihnen das Niveau der Volkswirtschaft niedriger als in den anderen sozialistischen Ländern.

Gen. Jugow bemerkte, dass die Rede Titos in Pula auch in Jugoslawien eine für die jugoslawische Führung ungünstige Resonanz gehabt hat.

Gen. Chruschtschow liest den Bericht des sowjetischen Botschafters in Jugoslawien über die Unterredungen mit Tito vor und sagt: Wie Sie sehen, argumentiert Tito in den Unterredungen richtig, macht aber alles genau umgekehrt.

Gen. Shiwkow teilt in diesem Zusammenhang mit, dass das ZK des BdKJ dem ZK der Kommunistischen Partei Bulgariens bereits den gesamten Briefwechsel zwischen dem ZK der KPdSU und dem ZK des BdKJ übergeben hat, darunter auch den letzten Brief.

Weiter sagte Gen. Chruschtschow, dass Tito und [Aleksandar] Ranković einen besseren Eindruck als die anderen Führer Jugoslawiens machen. Eine offen rechte Haltung beziehen [Koča] Popović und Todorović. Wohl noch schlimmer ist die Haltung von [Edvard] Kardelj. ${ }^{364}$ Sie kennen seine Vorlesung in Oslo. Seinerzeit haben wir mit Tito über diese Vorlesung gesprochen. Er sagte uns damals, er stimme in vieler Hinsicht auch nicht mit dieser Vorlesung überein, müsse aber die Situation berücksichtigen, in der diese Vorlesung gehalten wurde, insbesondere den Umstand, dass die Vorlesung noch vor der Normalisierung der sowjetischjugoslawischen Beziehungen gehalten wurde. Die folgenden Stellungnahmen Kardeljs haben aber gezeigt, dass er die gleichen Standpunkte [weiter] vertritt.

Im Zusammenhang damit bemerkt Gen. Tscherwenkow, dass nach seiner Ansicht Tito von beiden Seiten nimmt.

Als Antwort auf Gen. Tscherwenkow sagte Gen. Chruschtschow: Über Ihre Bewertung werde ich mich nicht mit Ihnen streiten.

Jetzt finden zwischen unserem Land und Jugoslawien Wirtschaftsverhandlungen statt. Wir haben die Fristen für die Kreditgewährung etwas verschoben, was

${ }^{364}$ Kardelj hatte als Theoretiker der jugoslawischen Partei das Konzept der Selbstverwaltung entwickelt, das sie nach dem Bruch mit Stalin diesem als Unterscheidungsmerkmal zum sowjetischen Sozialismus entgegenstellte und war generell in Belgrad entscheidend an der Formulierung der gegen die UdSSR gerichteten Linie beteiligt. In seiner Eigenschaft als Außenminister ab 1953 schloss er 1954 den Balkan-Pakt mit den NATO-Staaten Griechenland und Türkei, der nach der Übereinkunft zwischen Tito und der sowjetischen Führung am 2. Juni 1955 seine Bedeutung verlor. 
Tito sehr beunruhigt. In einem der Gespräche erklärte er, falls die Kreditierungstermine verschoben würden, könnten sie nicht das Aluminiumwerk bauen und die Produktion des Kunstdüngers zustande bringen, der dringend erforderlich sei für die Landwirtschaft, die bei ihnen im Niedergang begriffen ist. Sie können [dann] kein Aluminium ins Ausland verkaufen, und folglich werden sie keine Valuta haben, um im Ausland die von ihnen benötigten Güter einzukaufen. Wie war das denn? Sie spucken auf unser System und schmähen unsere Aufbaumethoden, und wir sollen ihnen ihr „fortschrittliches“ System finanzieren. Wir sollen uns vom Munde absparen, aber sie werden uns kritisieren.

Die Jugoslawen berufen sich oft darauf, dass sie einige Jahre lang eine isolierte Situation hatten, einer Blockade ausgesetzt waren und deswegen in wirtschaftlichen Schwierigkeiten sind. Dies ist dummes Zeug. Sie hatten keine Blockade auszuhalten, sondern erhielten Hilfe von den Amerikanern. Als wir in Jugoslawien waren, fiel uns auf, dass sie in einer Reihe von Orten gute Straßen haben. Die Sache ist die, dass die Jugoslawen das Geld aus den Erlösen des Verkaufs der Waren, die sie von den USA als Unterstützung erhalten haben, bei der Bank auf Kontokorrent einzahlen müssen und sie nur zu den Zwecken einsetzen dürfen, die ihnen von den Amerikanern genannt werden. Die Amerikaner sind natürlich nicht blöd und erlauben den Jugoslawen nicht, diese Gelder für die Entwicklung der Wirtschaft des Landes zu nutzen, sondern sie haben ihnen die Möglichkeit zur Verwendung dieser Mittel für Zwecke wie den Straßenbau gegeben im Wissen darum, dass vom Straßenbau her das Wirtschaftsniveau des Landes nicht besonders steigen wird.

Was die Blockade betrifft, der die Jugoslawen angeblich ausgesetzt waren, so haben wir nichts davon gesehen. Wir dagegen haben tatsächlich über viele Jahre hinweg in einer totalen Blockade gelebt. Und trotzdem, obwohl die Jugoslawen unseren Weg verurteilen, haben wir in 40 Jahren die Industrieproduktion im Land auf das 30 fache vergrößert.

Weiter sagte Gen. Chruschtschow, Tito hat in den Gesprächen erklärt, dass sie sich dafür entschieden haben, unsere Diskussion miteinander zu beenden, und den Wunsch nach einem Treffen mit uns auf hoher Ebene zum Ausdruck gebracht. Gen. Mikojan und ich haben im Gespräch mit Mićunović gesagt, dass wir mit dem Ende der Diskussion einverstanden sind, und wir haben unsere Zustimmung zu dem Treffen geäußert. Doch kurz danach erscheinen in der jugoslawischen Presse erneut Artikel, in denen die Sowjetunion beschimpft wird.

Die Jugoslawen versichern weiter, dass sie gegen die Blöcke sind und das Bestehen des sozialistischen Lagers nicht anerkennen wollen. Wir haben ihnen mehrfach gesagt, dass ihr zwar gegen die Blöcke und die Lager Stellung nehmt, selbst jedoch faktisch den Fuß in zwei Pakten habt. Folglich steht ihr nicht zwischen den Blöcken, sondern in einem der Blöcke. Da ihr über die Türkei mit dem Balkan-Pakt verbunden seid, nehmt ihr faktisch am Bagdad-Pakt ${ }^{365}$ und an der NATO teil.

365 Türkei und Irak hatten am 24. Februar 1955 ein - als Bagdad-Pakt bezeichnetes - Bündnis abgeschlossen, dem Großbritanniens am 5. April, Pakistan am 23. Mai und Iran am 3. November beitraten. 
Bei der Erörterung der ungarischen Ereignisse erklärte Tito, falls die Sowjetarmee nicht in Ungarn einmarschiert wäre, hätten sie, die Jugoslawen, ihre Armee einmarschieren lassen. In diesem Zusammenhang lachte Marschall [Georgij] Shukow ${ }^{366} \mathrm{zu}$ Recht, dass die ungarische Armee die jugoslawische Armee sicherlich zerschlagen hätte, deren Artilleriegeschütze von Pferden gezogen werden, während die ungarische Armee mit guten sowjetischen Panzern, Kanonen und Jagdflugzeugen ausgerüstet ist.

Weiter bemerkte Gen. Chruschtschow, dass der Konflikt in den Beziehungen der Jugoslawen zu den sozialistischen Ländern und den kommunistischen Parteien der kapitalistischen Welt, den Tito mit seiner Rede heraufbeschworen hatte, im jugoslawischen Volk nicht populär ist.

Danach wandte er sich der inneren Situation in der UdSSR zu. In der ausländischen Presse wird viel Geschrei darüber gemacht, dass es bei uns angeblich Demonstrationen, sogar fast bewaffnete Zusammenstöße usw. gegeben hat. Das ist alles Unsinn. So etwas hat es nicht gegeben. Insgesamt halten wir die Lage im Land für gut. Haben wir im Land antisowjetische Elemente und antisowjetische Auftritte? Natürlich gibt es das. In unserem Land lebt eine Bevölkerung von 200 Millionen, und es wäre erstaunlich, wenn wir keine antisowjetisch eingestellten Leute hätten. Dabei ist zu berücksichtigen, dass wir nach dem Tod Stalins Tausende aus den Gefängnissen entlassen haben, darunter auch Parteimitglieder. Die meisten aus den Gefängnissen entlassenen früheren Kommunisten wurden wieder in die Reihen der Partei aufgenommen. Natürlich musste man sie aus der Haft entlassen, doch einige von ihnen hätte man nicht wieder in die Partei aufnehmen sollen. Unter den Aufgenommenen hat es Leute gegeben, die seinerzeit zu Recht aus der Partei wegen ihres Kampfes gegen die Partei ausgeschlossen worden waren (Trotzkisten, Rechte usw.). Im Zusammenhang damit, dass man diese Leute aus den Gefängnissen entlassen und wieder in die Partei aufgenommen hat, ist es zu einer Belebung der gegen die Partei und die Sowjetunion gerichteten Tendenz gekommen, besonders in Leningrad. Einige der früheren Trotzkisten und Rechten haben erneut damit angefangen, ihre alten Ansichten zu predigen. Wir haben ein Schreiben an die Parteiorganisationen gerichtet und sie angewiesen, sich gegenüber den parteifeindlichen Stellungnahmen von Leuten, die gegen die Partei arbeiten, nicht liberal zu verhalten. Sie muss man ausschließen und, wenn sie ihre antisowjetische Tätigkeit fortsetzen, auch vor Gericht stellen. Es hat einzelne Auftritte von Studenten gegeben. In der bürgerlichen Presse wurde berichtet, dass wir im Zusammenhang damit angeblich auf breiter Front Repressivmaßnahmen angewendet haben. Das stimmt nicht. Wir haben keine Repressivmaßnahmen angewendet.

Die Landwirtschaft in unserem Land hat sich in den letzten Jahren bestens entwickelt. Im vergangenen Jahr wurde eine Ernte eingefahren, wie es sie noch nicht während des Bestehens der Sowjetmacht und auch nicht in der ganzen Geschichte Russlands gegeben hat.

Mithin ist unsere Lage beim Getreide sowie bei der Milch gut. Schlechter ist es um das Fleisch bestellt. Es stimmt zwar, dass wir im vergangenen Jahr einen ge- 
wissen Anstieg bei der Bereitstellung von Fleisch hatten, doch er reichte nicht aus. Jetzt beabsichtigen wir, strenge Maßnahmen zu ergreifen, um die Viehwirtschaft kräftig voranzubringen und den Fleischbedarf des Landes auf jeden Fall vollständig zu decken.

Wir haben eine Kostenanalyse für die landwirtschaftlichen Erzeugnisse angestellt, die der Staat von den Kolchosen und den Sowchosen erhält. Dabei stellte sich heraus, dass die Kosten für das Getreide, das wir von den Kolchosen ${ }^{367}$ beziehen, vor allem beim Einsatz der Maschinen- und Traktorenstationen sehr hoch ist. Im Landesdurchschnitt beträgt er etwa 61 Rubel. Die Sowchosen ${ }^{368}$ haben das Getreide zum Preis von 31 Rubel pro Zentner an den Staat abgegeben, einige Sowchosen sogar für 12 Rubel. Wir haben eine Kommission eingesetzt, die Vorschläge hinsichtlich der weiteren Bildung von Sowchosen auf der Basis von Kolchosen ausarbeitet. Wir haben die Absicht, die Sowchoswirtschaft auf diese Weise so weit zu entwickeln, dass wir schon in zwei Jahren von den Sowchosen jährlich mindestens 2 Milliarden Pud ${ }^{369}$ Getreide bekommen. Das ist genau das, was die Versorgung in unserem Land vollständig gewährleisten wird.

Ebenso ist es bei anderen Erzeugnissen der Landwirtschaft. Zum Beispiel sind bei den Sowchosen die Weintrauben zweimal billiger als bei den Kolchosen. Die von Sowchosen angebaute Baumwolle ist 30-35\% billiger als die Baumwolle, welche die Kolchosen liefern. Es ist zu sehen, dass die Kolchosbauern eine sehr starke Tendenz in Richtung Sowchosen haben. Das ist für sie vorteilhaft. Wenn sie Sowchos-Arbeiter werden, profitieren sie davon, weil sie einen monatlich garantierten Lohn, eine Rente im Alter und bei Invalidität, einen festgelegten Arbeitstag, einen Jahresurlaub und eine Reihe anderer Vorteile bekommen. Die baltischen Republiken verlangen, wir sollen alle Kolchosen dieser Republiken in Sowchosen umwandeln. Doch wir lassen uns jetzt nicht darauf ein, weil dort die Kolchosen sehr klein, die Bodenflächen klein sind und nirgends in großem Umfang die Mechanisierung durchführbar ist. Zudem ist der Boden dort schlecht. Derzeit gehen wir diesen Weg nicht, weil dafür große Kapitalinvestitionen erforderlich sind. Später wird sich das machen lassen.

Gen. Jugow sagt, dass in Bulgarien in letzter Zeit die Genossenschaften immer öfter fordern, ihnen staatlichen Boden zu überlassen. Die Staatswirtschaften arbeiten bei uns jetzt noch schlechter als die landwirtschaftlichen Produktionsgenossenschaften (TKZCh). ${ }^{370}$ Gen. Jugow bemerkt weiter, dass die jugoslawi-

367 Aufgrund der 1929 von Stalin verfügten Zwangskollektivierung war an die Stelle des auf eigenem Landbesitz beruhenden bäuerlichen Betriebs die landwirtschaftliche Kollektivwirtschaft (kollektivnoe chozjajstvo bzw. kolchos) mit gemeinsamem Eigentum an den Produktionsmittel, aber staatlichem Bodenbesitz getreten. Formal verwaltete sich diese Produktionsgenossenschaft selbst, unterstand aber faktisch der von der Partei eingesetzten Leitung. Sie hatten ein Ablieferungssoll zu staatlich festgesetzten Preisen zu erfüllen.

368 Ein Sowchos ("Sowjetwirtschaft“, sovetskoe chozjajstvo bzw. sovchoz) war ein landwirtschaftlicher Großbetrieb in Staatsbesitz, dessen Beschäftigte in aller Regel fest angestellt waren und feste monatliche Löhne erhielten.

3691 Pud $=16,38 \mathrm{~kg}$.

370 Die landwirtschaftlichen Produktionsgenossenschaften in den sozialistischen Ländern Mittelund Osteuropas entsprachen weithin den Kolchosen in der UdSSR, doch war der kollektiv genutzte Boden formal Privateigentum geblieben. 
sche Delegation, die in Bulgarien weilte, einzugestehen genötigt war, dass das Niveau der Landwirtschaft in Bulgarien weit höher ist als in Jugoslawien. In Jugoslawien liegen jetzt über 500000 ha Ackerland brach und werden nicht bestellt.

Gen. Chruschtschow sagt, als er noch einmal auf die Frage der Beziehungen zu Jugoslawien eingeht, dass wir, als wir den Bericht des sowjetischen Botschafters in der Sozialistischen Föderativen Republik Jugoslawien (SFRJ) über die Haltung Titos erhielten, froh über diese Information waren, weil wir dachten, dass die Jugoslawen in der Tat eine richtige Position beziehen, die Antwort aber, die sie schickten, erwies sich als komplett anders. Wir werden alle Möglichkeiten zu nutzen suchen, um eine Verbesserung der Beziehungen zu Jugoslawien $\mathrm{zu}$ erreichen, werden uns jedoch nicht auf prinzipielle Zugeständnisse einlassen.

Aufgezeichnet von I. Winogradow und M. Posolotin

Korrigiertes Stenogramm

Übersetzt aus dem russischen Originaltext

RGANI, fond 52, opis' 1, delo 550, Bl. 1-8

\section{Gespräch Chruschtschows mit einer Delegation französischer Radikalsozialisten ${ }^{371}$ unter Leitung des stellvertretenden Parteivorsitzenden und Fraktionsvorsitzenden, E[douard] Daladier, am 9. September 1957}

Nach den gegenseitigen Begrüßungen und dem Dank für den herzlichen und warmen Empfang, welcher der Delegation in der Sowjetunion bereitet wurde, erklärt Daladier, dass zwei Fragen die französische Öffentlichkeit erregen- das Problem der Abrüstung und die Zukunft Deutschlands. Er bittet darum, die Haltung der Sowjetunion zu diesen Fragen zu erläutern.

Chruschtschow: Sie wissen, dass sich die Sowjetunion seit vielen Jahren für ein Abkommen mit den Westmächten über die Abrüstung einsetzt. Doch bislang konnten keine Ergebnisse erzielt werden. Die Hauptursache dafür besteht darin, dass die westlichen Staaten noch nicht auf die sogenannte „Politik aus der Position der Stärke heraus“ verzichtet haben. Sie wissen, dass jede Reaktion eine Gegenreaktion hervorruft. Will man mit uns „aus der Position der Stärke heraus“ reden, wollen wir ebenfalls stark sein, um die Möglichkeit zum Widerstand gegen diese Politik zu haben. Auf diese Weise entsteht ein Wettbewerb bei der Entwicklung der Streitkräfte. Das ist das ganze Problem.

Wenn die Westmächte jetzt nicht auf die von ihnen betriebene „Politik aus der Position der Stärke heraus“ gegenüber der UdSSR verzichten, so wird es keine

371 Die französischen Radikalsozialisten waren keine sozialistische, sondern eine liberale Partei. 